\title{
CpG methylation patterns are associated with gene expression variation in osteosarcoma
}

\author{
QIANG WANG \\ Department of Orthopedics, The Second Affiliated Hospital of Harbin Medical University, \\ Harbin, Heilongjiang 150086, P.R. China
}

Received December 30, 2015; Accepted January 27, 2017

DOI: $10.3892 / \mathrm{mmr} .2017 .6635$

\begin{abstract}
Osteosarcoma is a common malignant tumor in childhood and adolescence (nearly 5\% of all cases of cancer in children), as well as a type of tumor with poor prognosis. However, the pathogenesis and molecular mechanisms of osteosarcoma remains to be elucidated. The aim of the current study was to determine the association between methylation and gene expression changes in osteosarcoma cell line. Microarray data were obtained from the Gene Expression Omnibus database (GSE36004). Genome-wide methylation status was determined in 19 different osteosarcoma cell lines and 6 normal controls. Differentially expressed genes (DEGs) were identified from cancer cells with genefilter package in $\mathrm{R}$ and differentially methylated sites were screened with CpGassoc package in R. Integrated gene expression with methylation profiles, genes differentially expressed and methylated, were obtained, and transcriptional regulatory network construction was performed. Functional annotation was performed for genes in the network using the DAVID online tool. Following integrated analysis, a total of 75 methylated sites were demonstrated to be localized at a transcription factor binding region. These sites may be bound by 83 transcription factors which will then alter the expression of 75 downstream DEGs. In the regulatory network, seizure related 6 homolog like 2 (SEZ6L2) had the highest degree of upregulation and was demonstrated to be regulated by 12 transcription factors. Furthermore, kin of IRRE like (KIRREL), centrosomal protein 72 (CEP72) and cyclin-dependent kinase 4 $(C D K 4)$ were also regulated by more than three transcription factors. Functional annotation revealed that the upregulated genes were primarily involved in the cell cycle pathway. Several differentially methylated sites were associated with upregulation of SEZ6L2, KIRREL, CEP72 and CDK4 and may
\end{abstract}

Correspondence to: Dr Qiang Wang, Department of Orthopedics, The Second Affiliated Hospital of Harbin Medical University, 157 Baojian Road, Harbin, Heilongjiang 150086, P.R. China

E-mail: wstrqh@163.com

Key words: osteosarcoma, methylation, transcriptional regulatory network have an important role in the pathogenesis of osteosarcomas through promotion of cell proliferation and metastasis.

\section{Introduction}

Osteosarcoma, which is most prevalent in childhood and adolescence, is an aggressive, malignant neoplasm that exhibits osteoblastic differentiation and produces malignant osteoid (1). Although the majority of patients are able to have limb surgery, various risk factors, including infection, complications of surgery and local tumor recurrence may induce the need for further surgery (2). In addition, although the 5-year survival rates of patients that received combined treatments, such as chemotherapy and surgery, may be as high as $70 \%$, rates in patients with lung metastasis remain unsatisfactory $(20-40 \%)(3,4)$. Thus, it is necessary to investigate the pathogenesis and molecular mechanisms of osteosarcoma in further depth to further advance treatment.

To date, various studies have investigated the molecular mechanism of osteosarcoma, including investigation of associated genes and pathways. Of those investigated, the retinoblastoma $(R B)$ gene and p53 gene are commonly implicated in the activation of metastatic osteosarcoma (5). Furthermore, it has been demonstrated that the integrity of the $R B$ pathway has an important role in tumor behavior, clinical progression and outcome in patients with osteosarcoma (6). Notably, restoration of $R B$ also corrected the activity of the $\mathrm{p} 53$ pathway in an aggressive osteosarcoma $(7,8)$. A previous study demonstrated that the methylation of heterozygous $R B$ and the $R B$ promoter have been exhibited in several patients with osteosarcoma (9). Previously, $\mathrm{CpG}$ island methylation, which is an epigenetic form of gene regulation that disturbs the function of tumor suppressor genes or oncogenes, has also been demonstrated to contribute to carcinogenesis (10). Skarn et al (11) demonstrated that methylation of $\mathrm{CpG}$ islands had an important role in the regulation of microRNA expression in osteosarcoma. However, the understanding of the epigenetic alterations implicated in osteosarcoma is currently limited. Therefore, the role of $\mathrm{CpG}$ methylation in the pathogenesis and progression of osteosarcoma remains to be defined in full.

A previous study made progress in the investigation of the molecular mechanisms of osteosarcoma dependent on methylation. Kresse et al (12) demonstrated the association between the copy number of DNA, mRNA expression and 
DNA methylation in osteosarcoma. Specifically, the present study focused on the effects of $\mathrm{CpG}$ island methylation in transcriptional regulation that contributes to the tumorigenesis of osteosarcoma. Based on the DNA methylation and gene expression profiles in osteosarcoma, differentially expressed genes (DEGs) with $\mathrm{CpG}$ methylation were identified, and the transcriptional regulatory relationship was analyzed by building a regulatory network. Functional annotation was also performed to investigate the biological role of abnormally expressed genes.

\section{Materials and methods}

Data source. Gene expression data of GSE36001 (ncbi. nlm.nih.gov/geo/query/acc.cgi?acc=GSE36001) and DNA methylation profiles of GSE36004 (ncbi.nlm.nih. gov/geo/query/acc.cgi?acc=GSE36004) were downloaded from the Gene Expression Omnibus (GEO) based on the platform of Illumina human-6 v.2.0 expression BeadChip (Illumina, Inc., San Diego, CA, USA) and Illumina HumanMethylation27 BeadChip (Illumina, Inc.) (12). In each of these datasets, 19 osteosarcoma cell lines were included, while two normal osteoblast cell lines (OB1 and OB2, two primary osteoblast cultures isolated from human calvaria of different donors) were purchased from ScienCell Research Laboratories (California, USA) (12) and four normal bone samples were included as controls. The following osteosarcoma cell lines were included: 143B; HAL; HOS; $\Omega$ IOR/OS9; IOR/OS10; IOR/OS14; IOR/OS15; IOR/OS18; IOR/MOS; IOR/SARG; KPD; MG-63; MHM; MNNG/HOS; OHS; OSA; Saos-2; U-2 OS; and ZK-58. Details of the origin of each cell line or bone sample involved in the datasets can be obtained via the web links provided for each dataset.

Data preprocessing. Raw data of all probes were normalized by the median method (13). Following normalization, the probe name was converted into a gene symbol based on the annotation information. If more than one probe mapped to one gene, an aggregate function in $\mathrm{R}$ (14) was performed to calculate the mean expression value for this gene. Probes with missing values were imputed with the nearest neighbor averaging method (15) of imputation (impute) package (version 1.0; https://bioconductor.org/packages/release/bioc/html/impute.html)in $\mathrm{R}$ (16). The DNA methylation data obtained from GEO was preprocessed with BeadStudio software (version 3.1) from Illumina, Inc. where the methylated locus for each sample with a missing value was removed.

Identification of DEGs in osteosarcoma cells. To identify DEGs between osteosarcoma cells and normal controls, one way analysis of variance (ANOVA) in the genefilter package of $\mathrm{R}$ was performed (15). P-values were generated using Benjamini-Hochberg (BH) multiple testing correction method and $\mathrm{P}<0.05$ was considered to indicate a statistically significant difference. The ratio of mean expression of normal and osteosarcoma cell groups was used to determine whether genes were up or downregulated.

Identification of disease-associated methylated regions $(D M R)$. To identify disease-associated $\mathrm{CpG}$ methylated sites in osteosarcoma cells, compared with normal control for disease-association analysis, $\mathrm{CpG}$ loci with beta values that were not significantly different between case and control were eliminated. Similarly, all CpG loci on the X, Y and mitochondrial chromosomes were removed. Subsequently, the CpGassoc package of $\mathrm{R}(17)$ was used for disease-association analysis, which is designed to investigate the association between methylation at $\mathrm{CpG}$ loci across the genome and a phenotype of interest. CpGassoc algorithm of $\mathrm{R}$ package was used to determine the association between $\mathrm{CpG}$ loci and osteosarcoma. In addition, CpGassoc can also be used to create quantile-quantile plots, manhattan plots and scatterplots for individual $\mathrm{CpG}$ sites. $\mathrm{BH}$ multiple testing correction was used to estimate the false discovery rate (FDR) in disease-associated analysis. FDR $\leq 0.05$ was chosen as the threshold.

Integration analysis of gene expression and methylation profiles. To investigate the association between methylation and gene expression in osteosarcoma cell lines, methylation data was measured on $\pm 2 \mathrm{~kb}$ genomic regions around the transcriptional start sites (TSS) of each gene. The obtained genes were differentially methylated. Integration analysis was performed to identify genes where there was an overlap between differential expression between control and osteosarcoma, and the presence of methylation. In order to investigate the influence of methylation on gene expression, the transcription factor binding sites were searched within the UCSC database (18). Subsequently, methylated DEGs in transcription factor binding regions in osteosarcoma cells were screened.

Construction of transcriptional regulatory networks. Based on the transcription factor and target gene information provided by the UCSC database, transcriptional regulatory networks were constructed and further visualized by Cytoscape software (version 2.8.0; www.cytoscape.org) (19). In the network, the node degree was calculated by igraph package in $\mathrm{R}(20)$.

Functional annotation of target genes of transcription factors. To understand the biological roles of target genes of the transcription factors blocked by DNA methylation, gene ontology (GO) function and pathway enrichment analysis was performed by DAVID (database for annotation, visualization, and integrated discovery) online tool (21). $\mathrm{P}<0.05$ was considered to indicate a statistically significant difference.

\section{Results}

Identification of DEGs in osteosarcoma cells. Following preprocessing of methylation profile data, a total of 20,006 methylation sites were identified from the 25 samples Similarly, a total of 24,214 genes were identified from 25 samples following preprocessing of expression profile data. By applying ANOVA, a total of 6,419 DEGs were identified in osteosarcoma cells compared with normal control, including 3,236 upregulated and 3,183 downregulated genes.

Identification of DMRs. To identify differentially methylated genes, the present study performed three types of analyses. Initially preprocessing of raw methylation data was performed and 20,006 methylated sites were identified in the 25 samples 


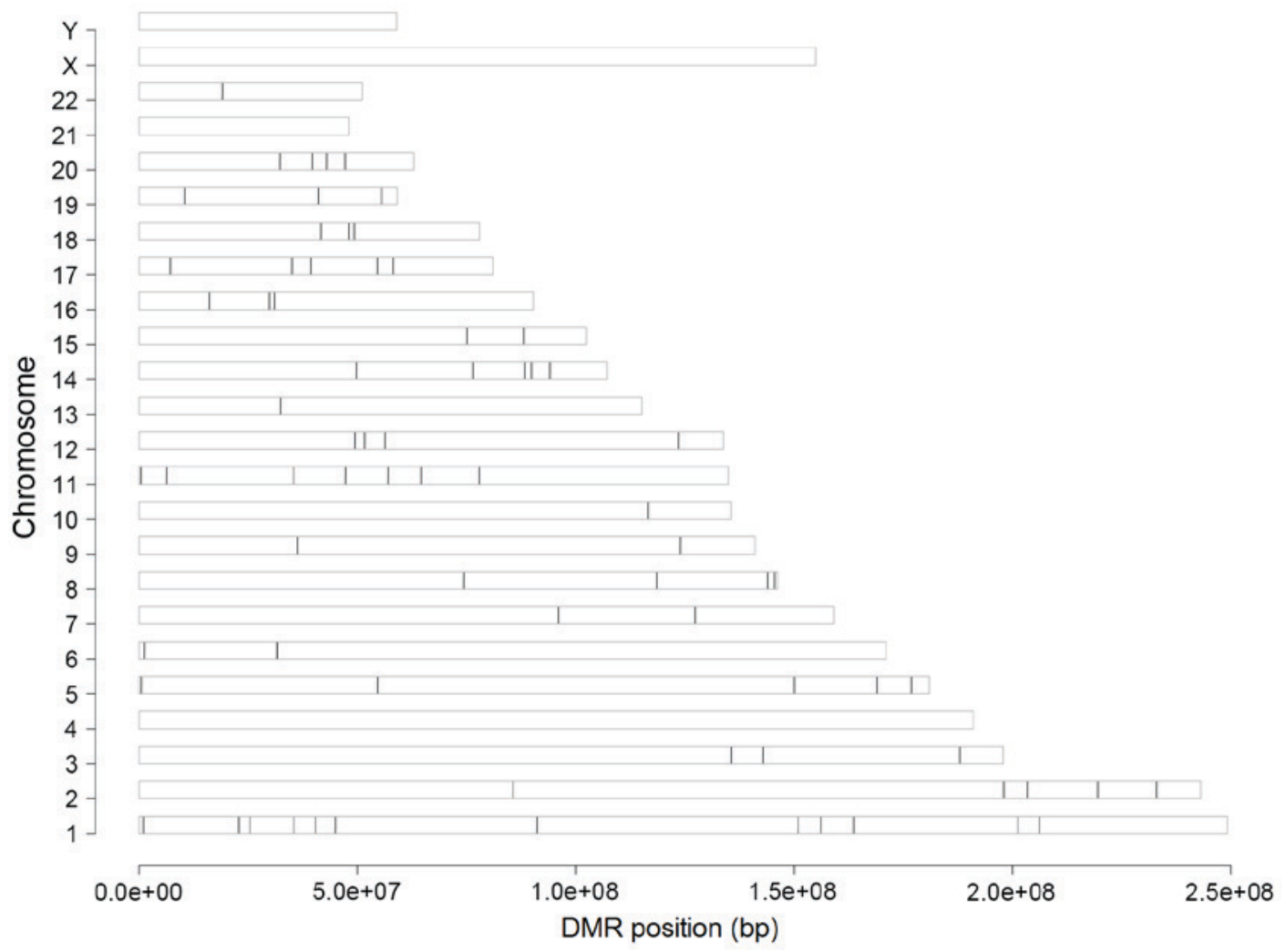

Figure 1. Distribution of DMRs on chromosomes. The y axis indicates chromosome location and the $\mathrm{x}$ axis indicates the position of DMRs. All DMRs located on the $\mathrm{X}, \mathrm{Y}$ and mitochondrial chromosomes were removed. DMRs, disease-associated methylated regions.

included in the dataset. Subsequently, methylated sites that were located on the $\mathrm{X}, \mathrm{Y}$ and mitochondrial chromosomes and imputation analysis was performed; as a result, 5,921 sites were eliminated from further analysis. Finally, following disease-associated analysis, 13,750 differentially methylated genes located in different chromosome regions, with an FDR $<0.05$, were identified in osteosarcoma cells (Fig. 1).

Integration analysis of DEGs and differentially methylated genes. Integration analysis indicated that 3,625 genes were differentially expressed in osteosarcoma and control, and also methylated around their TSS. Based on the UCSC database for transcription factors, a total of 75 methylated sites were located in the transcription factor binding regions, which may affect 83 transcription factors and 75 downstream target genes.

Construction of transcription regulatory network. Based on different types of regulation, a transcription regulatory network was constructed, which included 83 transcription factors and 75 downstream target genes (Fig. 2). In the network, there were 158 nodes and 129 edges. Of the genes in the network, the overexpressed gene seizure related 6 homolog like 2 (SEZ6L2) had the highest degree. It was observed that SEZ6L2 was regulated by 12 transcription factors, including signal transducer and activator of transcription 3 (STAT3) and early growth response 1-3 (EGR1-3). Additionally, the transcription factor myocyte enhancer factor 2 ( $M E F 2$ ), which had a higher degree compared with other transcription factors, regulated seven downstream targets, including the upregulated replication factor C (activator 1) 4, cyclin-dependent kinase 4 (CDK4) and chromodomain helicase DNA binding protein 6 genes, and downregulated the chromosome 14 open reading frame 102 gene. Similarly, the upregulated kin of IRRE like gene (KIRREL) was regulated by seven different transcription factors. Transcription factor paired box $5(P A X 5)$ was indicated to be involved in the regulation of three up and two downregulated genes in the network. Upregulated genes, centrosomal protein 72 (CEP72), block of proliferation 1 (BOP1) and TruB pseudouridine synthase family member 1 , were regulated by a different set of three transcription factors: STAT3, MEF2 and adaptor-related protein complex 1 (AP1).

Functional annotation of target genes of transcription factors. GO functional enrichment analysis was performed for 46 upregulated and 29 downregulated genes. The top five enriched categories are listed in Tables I and II. The upregulated genes were predominantly enriched in the GO terms of cell cycle, non-coding RNA metabolic process and the maturation of large subunit ribosomal RNA (rRNA) from tricistronic rRNA transcript. In addition, downregulated genes were predominantly involved in the inflammatory response, regulation of the humoral immune response and response to wounding.

\section{Discussion}

Osteosarcoma is the most common histological form of primary bone cancer. To analyze the effects of genome-wide changes in gene expression and DNA methylation in osteosarcoma cell lines, the present study identified 75 significantly 


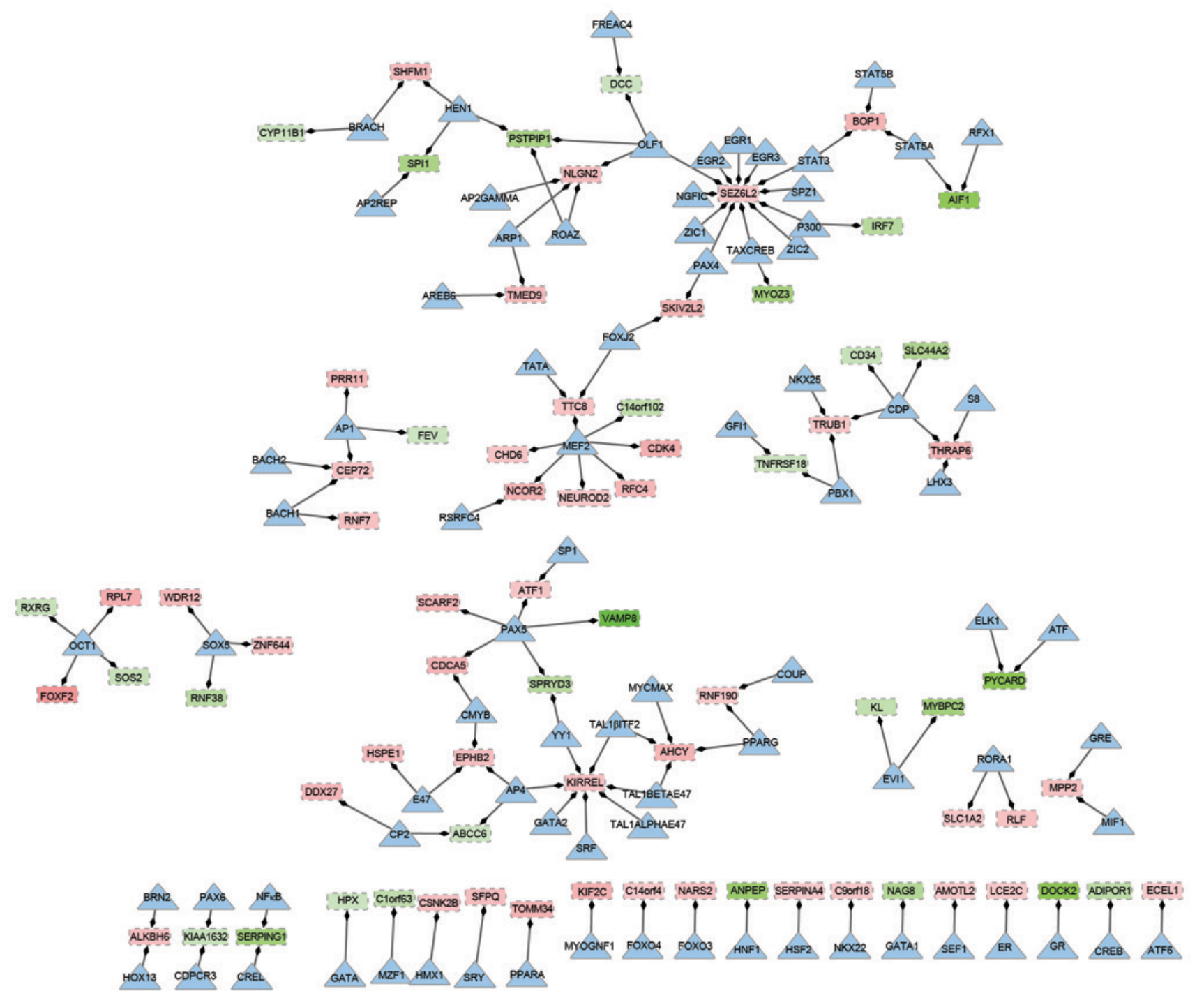

Figure 2. Transcriptional regulatory network. Blue triangle, transcription factor; red rectangle, upregulated gene; green rectangle, downregulated gene.

methylated genes, which included 46 genes that were upregulated (including SEZ6L2, KIRREL, CEP72, BOP1 and CDK4) and 29 genes that were downregulated in osteosarcoma cell lines compared with the normal controls. These genes were regulated by 83 transcription factors, including MEF2 and PAX5.

SEZ6L2 encodes a seizure-associated protein with an N-terminal signal peptide that is located on the cell surface. In the present study, this gene was demonstrated to be regulated by various transcription factors, including STAT3, EGRI and PAX4. It has been previously demonstrated that STAT3 upregulates vascular endothelial growth factor expression and contributes to tumor angiogenesis (22), which means it is widely regarded as a promising target for cancer treatment. In addition, the STAT3 inhibitor, CDDO-Me, inhibited the development of osteosarcoma cell lines and also induced apoptosis (23). Furthermore, it was demonstrated that microRNA (miR)-125b downregulated the expression of STAT3, which suppressed the migration and proliferation of osteosarcoma cells (24). In addition, a previous study demonstrated that, by upregulating EGR1, chemotherapy downregulated the activity of urokinase and prevented osteosarcoma cell invasion (25).
Additionally, another member of EGR family, EGR2, was suppressed by miR-20a, which promoted the cell cycle and proliferation of human osteosarcoma cells (26). Based on these previous results, SEZ6L2 may modulate the cell cycle and metastasis of osteosarcoma through regulation by STAT3, EGRI and PAX4.

KIRREL, also known as NEPH1, is a nephrin-associated member of the immunoglobulin superfamily that is involved in cell-cell interaction and somatic cell fusion during embryonic development $(27,28)$. Notably, it is thought that somatic cell fusion may be a mechanism that contributes to cancer metastasis and chemotherapy resistance $(29,30)$. KIRREL has been demonstrated to be differentially hyper-methylated in primary malignant adrenocortical samples compared with benign samples (31). In the present study, it was identified that KIRREL was regulated by various transcription factors, including AP4, GATA binding protein 2 (GATA2) and serum response factor (SRF). AP4 induced the expression of CDK2, which subsequently regulated the proliferation of osteosarcoma cell lines (32). SRF was previously demonstrated to be involved in the mitogen-activated protein kinase cascade signaling pathway 
Table I. GO function and pathway enrichment analysis of upregulated genes in the transcriptional regulatory network.

Term

\begin{tabular}{|c|c|c|c|}
\hline ID & Name & Count & P-value \\
\hline \multicolumn{4}{|c|}{ REACTOME_PATHWAY } \\
\hline REACT_152 & Cell cycle, mitotic & 4 & 0.028263722 \\
\hline \multicolumn{4}{|c|}{ GOTERM_BP_FAT } \\
\hline GO:0034660 & ncRNA metabolic process Maturation of LSU-rRNA & 5 & 0.002014149 \\
\hline GO:0000463 & $\begin{array}{l}\text { from tricistronic rRNA transcript (small subunit-rRNA, } \\
5.8 \mathrm{~S} \text { rRNA and LSU-rRNA) }\end{array}$ & 2 & 0.007080142 \\
\hline GO:0000470 & Maturation of LSU-rRNA & 2 & 0.007080142 \\
\hline GO:0006396 & RNA processing & 6 & 0.008649471 \\
\hline GO:0034470 & ncRNA processing & 4 & 0.009603024 \\
\hline \multicolumn{4}{|c|}{ GOTERM_CC_FAT } \\
\hline GO:0070013 & Intracellular organelle lumen & 12 & 0.002136088 \\
\hline GO:0043233 & Organelle lumen & 12 & 0.002571635 \\
\hline GO:0031974 & Membrane-enclosed lumen & 12 & 0.00301231 \\
\hline GO:0005654 & Nucleoplasm & 8 & 0.004426136 \\
\hline GO:0031981 & Nuclear lumen & 10 & 0.006006103 \\
\hline \multicolumn{4}{|c|}{ GOTERM_MF_FAT } \\
\hline GO:0005524 & ATP binding & 9 & 0.019812363 \\
\hline GO:0032559 & Adenyl ribonucleotide binding & 9 & 0.021338813 \\
\hline GO:0008026 & ATP-dependent helicase activity & 3 & 0.022750064 \\
\hline GO:0070035 & Purine nucleotide triphosphate-dependent helicase activity & 3 & 0.022750064 \\
\hline GO:0042623 & ATPase activity, coupled & 4 & 0.026532158 \\
\hline
\end{tabular}

REACT, reactome; GO, gene ontology; BP, biological process; CC, cellular component; MF, molecular function; ncRNA, non-coding RNA; LSU-rRNA, large subunit ribosomal RNA.

Table II. Functional annotation of downregulated genes in the transcriptional regulatory network.

\begin{tabular}{|c|c|c|c|}
\hline \multicolumn{2}{|r|}{ Term } & \multirow[b]{2}{*}{ Count } & \multirow[b]{2}{*}{ P-value } \\
\hline ID & Name & & \\
\hline \multicolumn{4}{|c|}{ GOTERM_BP_FAT } \\
\hline GO:0006954 & Inflammatory response & 4 & 0.01325742 \\
\hline GO:0002920 & Regulation of humoral immune response & 2 & 0.018477257 \\
\hline GO:0009611 & Response to wounding & 4 & 0.047076136 \\
\hline \multicolumn{4}{|c|}{ GOTERM_MF_FAT } \\
\hline GO:0005496 & Steroid binding & 3 & 0.004729209 \\
\hline
\end{tabular}

GO, gene ontology; BP, biological process; MF, molecular function.

in human osteosarcoma cells (33). Furthermore, it has been demonstrated that GATA2 is required for proliferation in various cancer cell types (34). Thus, differential expression of KIRREL may be regulated by methylation and transcription factors that promote tumor development. In addition, $C E P 72, C D K 4$ and $B O P 1$ were differentially methylated and regulated by transcription factors, including STAT3, MEF2 and AP1. CEP72 encodes a protein that is a member of the leucine-rich-repeat superfamily. CEP72 has been identified as possessing a high incidence of genomic copy number changes in the 5 p15.33 region in patients with non-small cell lung cancer (35). As osteosarcoma has a high tendency for metastatic spread and predominantly arises in the lungs, CEP72 may have a key role in cancer metastasis. In addition, it was previously demonstrated that CEP72 was regulated by AP1, which is involved in the ERK signaling pathway (36). It is well established that CDKs are essential drivers of cell cycle progression and are commonly dysregulated during 
tumorigenesis (37). CDK4 and other CDK inhibitors have been identified as a class of promising anticancer agents in cancer treatment (38). Furthermore, regulated transcription factors STAT3 and AP1 have been demonstrated to be involved in cancer development and progression via promotion of the cell cycle $(39,40)$. The functional annotation performed in the present study was consistent with a previous study about the enriched pathway of $C E P 72$ and $C D K 4$ (41).

In conclusion, methylation of SEZ6L2, KIRREL, CEP72 and $C D K 4$ may have an important role in the pathogenesis of osteosarcomas through promotion of cell proliferation and metastasis. However, further study into the results is required and may provide further insight into the molecular mechanism of osteosarcoma. In addition, further experiments, including western blot analysis and reverse transcription-polymerase chain reaction will be performed in the future to validate the changes in gene expression.

\section{References}

1. Luetke A, Meyers PA, Lewis I and Juergens H: Osteosarcoma treatment-where do we stand? A state of the art review. Cancer Treat Rev 40: 523-532, 2014.

2. Jaffe N, Bruland OS and Bielack S (eds.): Pediatric and adolescent osteosarcoma. Springer Science \& Business Media, 2010.

3. Buddingh EP, Anninga JK, Versteegh MI, Taminiau AH, Egeler RM, van Rijswijk CS, Hogendoorn PC, Lankester AC and Gelderblom H: Prognostic factors in pulmonary metastasized high-grade osteosarcoma. Pediatr Blood Cancer 54 216-221, 2010.

4. Lewis IJ, Nooij MA, Whelan J, Sydes MR, Grimer R, Hogendoorn PC, Memon MA, Weeden S, Uscinska BM, van Glabbeke M, et al: Improvement in histologic response but not survival in osteosarcoma patients treated with intensified chemotherapy: A randomized phase III trial of the European Osteosarcoma Intergroup. J Natl Cancer Inst 99: 112-128, 2007.

5. Berman SD, Calo E, Landman AS, Danielian PS, Miller ES West JC, Fonhoue BD, Caron A, Bronson R, Bouxsein ML, et al Metastatic osteosarcoma induced by inactivation of $\mathrm{Rb}$ and p53 in the osteoblast lineage. Proc Natl Acad Sci USA 105: 11851-11856, 2008.

6. Scott MC, Sarver AL, Phan F, Gupta R, Thayanithy V, Subramanian S and Modiano F: Abstract B19: RB function as a central component of osteosarcoma behavior: A comparative assessment in dogs and humans. Molecular Cancer Res 12: B19, 2014.

7. Ternovoi VV, Curiel DT, Smith BF and Siegal GP: Adenovirus-mediated p53 tumor suppressor gene therapy of osteosarcoma. Lab Invest 86: 748-766, 2006.

8. Scott MC, Sarver AL, Tomiyasu H, Cornax I, Van Etten J, Varshney J, O'Sullivan MG, Subramanian S and Modiano JF: Aberrant retinoblastoma (RB)-E2F transcriptional regulation defines molecular phenotypes of osteosarcoma. J Biol Chem 290: 28070-28083, 2015.

9. Patiño-García A, Piñeiro ES, Díez MZ, Iturriagagoitia LG, Klüssmann FA and Ariznabarreta LS: Genetic and epigenetic alterations of the cell cycle regulators and tumor suppressor genes in pediatric osteosarcomas. J Pediatr Hematol Oncol 25 362-367, 2003

10. Cui J, Wang W, Li Z, Zhang Z, Wu B and Zeng L: Epigenetic changes in osteosarcoma. Bull Cancer 98: E62-E68, 2011.

11. Skarn M, Namlos HM, Ahmed D, Lind GE, Meza-Zepeda LA and Myklebost O: Epigenetic regulation of miRNA expression in osteosarcoma. Cancer Res 72: 197, 2012.

12. Kresse SH, Rydbeck H, Skarn M, Skårn M, Namløs HM, Barragan-Polania AH, Cleton-Jansen AM, Serra M, Liestøl K, Hogendoorn PC, et al: Integrative analysis reveals relationships of genetic and epigenetic alterations in osteosarcoma. PLoS One 7: e48262, 2012.

13. Irizarry RA, Hobbs B, Collin F, Beazer-Barclay YD, Antonellis KJ, Scherf U and Speed TP: Exploration, normalization, and summaries of high density oligonucleotide array probe level data. Biostatistics 4: 249-264, 2003.
14. Wang J, Xie G, Singh M, Ghanbarian AT, Raskó T, Szvetnik A, Cai H, Besser D, Prigione A, Fuchs NV, et al: Primate-specific endogenous retrovirus-driven transcription defines naive-like stem cells. Nature 516: 405-409, 2014.

15. Altman NS: An introduction to kernel and nearest-neighbor nonparametric regression. The American Statistician 46: 175-185, 1992.

16. Hastie T, Tibshirani R, Narasimhan B and Chu G: Impute: imputation for microarray data. Bioinformatics 17: 520-525, 2001.

17. Barfield RT, Kilaru V, Smith AK and Conneely KN: CpGassoc: An $\mathrm{R}$ function for analysis of DNA methylation microarray data. Bioinformatics 28: 1280-1281, 2012.

18. Meyer LR, Zweig AS, Hinrichs AS, Karolchik D, Kuhn RM, Wong M, Sloan CA, Rosenbloom KR, Roe G, Rhead B, et al: The UCSC Genome Browser database: Extensions and updates 2013. Nucleic Acids Res 41: D64-D69, 2013

19. Kohl M, Wiese S and Warscheid B: Cytoscape: Software for visualization and analysis of biological networks. In: Data Mining in Proteomics. Springer, pp291-303, 2011.

20. Csardi G and Nepusz T: The igraph software package for complex network research. Inter J Complex Systems 1695, 2006.

21. Dennis G Jr, Sherman BT, Hosack DA, Yang J, Gao W, Lane HC and Lempicki RA: DAVID: Database for annotation, visualization, and integrated discovery. Genome Biol 4: P3, 2003.

22. Wei D, Le X, Zheng L, Wang L, Frey JA, Gao AC, Peng Z, Huang S, Xiong HQ, Abbruzzese JL and Xie K: Stat 3 activation regulates the expression of vascular endothelial growth factor and human pancreatic cancer angiogenesis and metastasis. Oncogene 22: 319-329, 2003.

23. Ryu K, Choy E, Yang C, Susa M, Hornicek FJ, Mankin H and Duan Z: Activation of signal transducer and activator of transcription 3 (Stat3) pathway in osteosarcoma cells and overexpression of phosphorylated-Stat 3 correlates with poor prognosis. J Orthop Res 28: 971-978, 2010

24. Liu LH, Li H, Li JP, Zhong H, Zhang HC, Chen J and Xiao T: miR-125b suppresses the proliferation and migration of osteosarcoma cells through down-regulation of STAT3. Biochem Biophys Res Commun 416: 31-38, 2011.

25. Matsunoshita $Y$, Ijiri K, Ishidou Y, Nagano S, Yamamoto T, Nagao H, Komiya S and Setoguchi T: Suppression of osteosarcoma cell invasion by chemotherapy is mediated by urokinase plasminogen activator activity via up-regulation of EGR1. PLoS One 6: e16234, 2011.

26. Zhuo W, Ge W, Meng G, Jia S, Zhou X and Liu J: MicroRNA-20a promotes the proliferation and cell cycle of human osteosarcoma cells by suppressing early growth response 2 expression. Mol Med Rep 12: 4989-4994, 2015.

27. Sellin L, Huber TB, Gerke P, Quack I, Pavenstädt H and Walz G: NEPH1 defines a novel family of podocin interacting proteins. FASEB J 17: 115-117, 2003.

28. Durcan PJ, Al-Shanti N and Stewart CE: Identification and characterization of novel Kirrel isoform during myogenesis. Physiol Rep 1: e00044, 2013.

29. Duelli D and Lazebnik Y: Cell fusion: A hidden enemy? Cancer Cell 3: 445-448, 2003.

30. Pawelek JM and Chakraborty AK: The cancer cell-leukocyte fusion theory of metastasis. Adv Cancer Res 101: 397-444, 2008.

31. Rechache NS, Wang Y, Stevenson HS, Killian JK, Edelman DC, Merino M, Zhang L, Nilubol N, Stratakis CA, Meltzer PS and Kebebew E: DNA methylation profiling identifies global methylation differences and markers of adrenocortical tumors. J Clin Endocrinol Metab 97: E1004-E1013, 2012.

32. Jackstadt R, Röh S, Neumann J, Jung P, Hoffmann R, Horst D, Berens C, Bornkamm GW, Kirchner T, Menssen A and Hermeking H: AP4 is a mediator of epithelial-mesenchymal transition and metastasis in colorectal cancer. J Exp Med 210: 1331-1350, 2013.

33. Bébien M, Salinas S, Becamel C, Richard V, Linares L and Hipskind RA: Immediate-early gene induction by the stresses anisomycin and arsenite in human osteosarcoma cells involves MAPK cascade signaling to Elk-1, CREB and SRF. Oncogene 22: 1836-1847, 2003

34. Tsai FY and Orkin SH: Transcription factor GATA-2 is required for proliferation/survival of early hematopoietic cells and mast cell formation, but not for erythroid and myeloid terminal differentiation. Blood 89: 3636-3643, 1997.

35. Kang JU, Koo SH, Kwon KC, Park JW and Kim JM: Gain at chromosomal region 5p15.33, containing TERT, is the most frequent genetic event in early stages of non-small cell lung cancer. Cancer Genet Cytogenet 182: 1-11, 2008. 
36. Cho HJ, Kang JH, Kwak JY, Lee TS, Lee IS, Park NG, Nakajima H, Magae J and Chang YC: Ascofuranone suppresses PMA-mediated matrix metalloproteinase-9 gene activation through the Ras/Raf/MEK/ERK-and Ap1-dependent mechanisms. Carcinogenesis 28: 1104-1110, 2007.

37. Trovesi C, Manfrini N, Falcettoni M and Longhese MP: Regulation of the DNA damage response by cyclin-dependent kinases. J Mol Biol 425: 4756-4766, 2013.

38. Finn RS, Crown JP, Lang I, Boer K, Bondarenko IM, Kulyk SO Etti J, Patel R, Pinter T, Schmidt M, et al: Results of a randomized phase 2 study of PD 0332991, a cyclin-dependent kinase (CDK) $4 / 6$ inhibitor, in combination with letrozole vs. letrozole alone for first-line treatment of ER+/HER2-advanced breast cancer (BC). Cancer Res 72: S1-S6, 2012.
39. Grivennikov SI and Karin M: Dangerous liaisons: STAT3 and NF-kappaB collaboration and crosstalk in cancer. Cytokine Growth Factor Rev 21: 11-19, 2010

40. Ishdorj G, Johnston JB and Gibson SB: Cucurbitacin-I (JSI-124) activates the JNK/c-Jun signaling pathway independent of apoptosis and cell cycle arrest in B leukemic cells. BMC Cancer 11: $268,2011$.

41. Oshimori N, Li X, Ohsugi M and Yamamoto T: Cep72 regulates the localization of key centrosomal proteins and proper bipolar spindle formation. EMBO J 28: 2066-2076, 2009. 